\title{
Lower limb movement intention recognition for rehabilitation robot aided with projected recurrent neural network
}

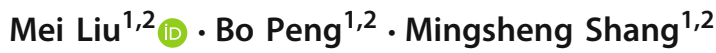

Received: 10 December 2020 / Accepted: 17 March 2021 / Published online: 30 March 2021

(c) The Author(s) 2021

\begin{abstract}
For the lower limb rehabilitation robot, how to better realize intention recognition is the key issue in the practical application. Recognition of the patient's movement intention is a challenging research work, which needs to be studied from the shallow to the deep. Specifically, it is necessary to ensure that the movement intention of the normal person can be accurately recognized, and then improve the model to realize the recognition of the movement intention of the patients. Therefore, before studying the patient's movement intention, it is essential to consider the normal person first, which is also for safety considerations. In recent years, a new Hill-based muscle model has been demonstrated to be capable of directly estimating the joint angle intention in an open-loop form. On this basis, by introducing a recurrent neural network (RNN), the whole prediction process can achieve more accuracy in a closed-loop form. However, for the traditional RNN algorithms, the activation function must be convex, which brings some limitations to the solution of practical problems. Especially, when the convergence speed of the traditional RNN model is limited in the practical applications, as the error continues to decrease, the convergence performance of the traditional RNN model will be greatly affected. To this end, a projected recurrent neural network (PRNN) model is proposed, which relaxes the condition of the convex function and can be used in the saturation constraint case. In addition, the corresponding theoretical proof is given, and the PRNN method with saturation constraint has been successfully applied in the experiment of intention recognition of lower limb movement compared with the traditional RNN model.
\end{abstract}

Keywords Projected recurrent neural network (PRNN) · Lower limb movement $\cdot$ Intention recognition

\section{Introduction}

In robot control field, it has become a research hotspot to realize the high-precision control of robots and to further achieve the human-computer interaction (HCI) [1-6]. As a core element in the process of HCI, various methods concerning human intention recognition have been researched by many experts. For example, a probabilistic plan recognition algorithm based on plan tree grammars is explored in [7].

\section{Mei Liu \\ liumeisysu@qq.com \\ Bo Peng \\ pengbo@ cigit.ac.cn}

Mingsheng Shang

msshang@cigit.ac.cn

1 Chongqing Key Laboratory of Big Data and Intelligent Computing, Chongqing Institute of Green and Intelligent Technology, Chinese Academy of Sciences, Chongqing 400714, China

2 University of Chinese Academy of Sciences, Beijing 100049, China
The role of intention recognition in the evolution of cooperative behavior is discussed in [8]. A logic-based approach is developed for intention recognition [9]. However, the above methods are mainly used to predict the macro intentions of human body, and cannot accurately predict a specific movement in real time. As a significant input biological signal, surface electromyography (sEMG) signal is widely used in the intention recognition of the rehabilitation robot, which contains a lot of information about the body limbs such as muscle strength and joint moment arm [10-12]. How to extract these behavioral information from sEMG signal effectively and estimate the next human body motion state has become a vital research direction. Owing to the important role of intention recognition in our life, many experts have established various methods for this nonlinear system.

In recent years, Hill-based muscle model (HMM) [13] has been widely applied in the domain of the motion intention recognition as a classic muscle model, which can well explain the relationship between sEMG signals and the muscle strength. However, on account of the complexity of the model, most of the physiological parameters need to be esti- 
mated, which leads to the accumulation of errors in the process of motion prediction $[14,15]$. Therefore, it cannot be directly applied to rehabilitation robot. Based on HMM, Han et al. establish a closed-loop prediction model combined with Kalman filter of the upper limb to effectively eliminate the error accumulation in the prediction process [16]. Nevertheless, there are few motion prediction methods for lower limb joints, so it is necessary to carry out relevant research.

With the development of artificial intelligence and industrial control, more and more intelligent algorithms have emerged [17-21]. Consequently, neural networks have been increasingly popular with many applications and investigated widely for the excellent calculation power and relative robustness [22-25]. Recently, recurrent neural network (RNN) has attracted considerable attention as candidates for novel computational systems, which can be used for solving time-varying nonlinear equations [26]. Besides, RNN is often designed as an ordinary differential equation for zeroing finding in the field of industrial system tracking control [27-30]. However, in actual industrial control, the convergence rate of the controller for errors is often limited by the influence of hardware devices, which causes the convergence performance of the traditional RNN with convex constraints to be seriously affected as the error decays. To remedy the weakness of traditional RNN model in the case of convergence rate limited, a PRNN model is proposed in this paper, which can be of saturation and non-convexity different from that should be unbounded and convex described in the traditional RNN model. The corresponding numerical experiments confirm the superiority of the PRNN model with saturation constraints over the traditional RNN model in the case of limited convergence rate. In addition, combined with HMM, this paper innovatively applies the PRNN model with saturation constraints and the traditional RNN model to the intention recognition of lower limb movement to build a closed-loop recognition framework. By means of constantly updating the control law, the whole recognition process avoids the error accumulation and achieves the continuous accurate prediction of lower limb movement intention. Moreover, the experimental results also show that under the condition of limited convergence rate, the PRNN model with saturation constraints can better estimate the intention of lower limb movement compared with the traditional RNN model.

The rest of the article is arranged as follows. In Sect. 2, the design formula of PRNN model is presented. In Sect. 3, the mathematical derivation of the convergence and robustness of PRNN model is provided. For the traditional RNN model and the PRNN model with saturation constraints, comparative simulation experiments are carried out in Sect. 4. The process of intention recognition of lower limb and the recognition principle combining with PRNN model are given in Sect. 5. Based on the specific saturation activation function and related lower limb parameters, the experimental sim- ulations of lower limb intention recognition are offered in Sect. 6. Section 7 summarizes the research course of this paper. Finally, the main contributions made by this paper are pointed out as below.

1. This paper compares the convergence and robustness of the PRNN model with saturation constraints and the traditional RNN model when the convergence rate is limited.

2. Through the Lyapunov stability theorem, strict theoretical proofs and analysis are given for the convergence and robustness of the PRNN model.

3. Under the condition that the convergence rate is limited, numerical simulation experiments are performed to demonstrate the superiority of PRNN model with saturation constraints compared with the traditional RNN model.

4. Regarding the motion intention recognition of lower limb rehabilitation robot, based on the state-space HMM (SSHMM), a new closed control method implanting PRNN model with saturation constraints is proposed. Besides, compared with the traditional RNN model, simulation experiments are conducted to verify the superior convergence performance and robustness of PRNN model with saturation constraints when the convergence rate is limited.

\section{Problem formulation and solution}

Combined with the general form of time-varying nonlinear equations, the formula derivation of the proposed PRNN model is given in this section, and the traditional RNN model is also presented.

\section{Problem formulation}

Most robot control problems can be converted into the following time-varying nonlinear equations [31-33]:

$y(\mathbf{x}(t), t)=\mathbf{0} \in \mathbb{R}^{m}, t \in[0, \infty)$,

where $y(\cdot)$ represents a nonlinear mapping. Naturally, the corresponding robot control solution is converted to the solving of $\mathbf{x}(t)$ at any time instant $t \in[0, \infty)$, and only when the actual solution $\mathbf{x}(t)$ reaches the theoretical solution $\mathbf{x}^{*}(t)$, can the right side of equation (1) be zero, that is, $y\left(\mathbf{x}^{*}(t), t\right)=\mathbf{0}$.

\section{Continuous-time PRNN model}

In general, for the solution of nonlinear equations, since there is no exact solution formula, the commonly used RNN model [27-29] is to iterate the error function, thus approximating 
Fig. 1 Block diagram of implementing the proposed PRNN model (5) from control perspective

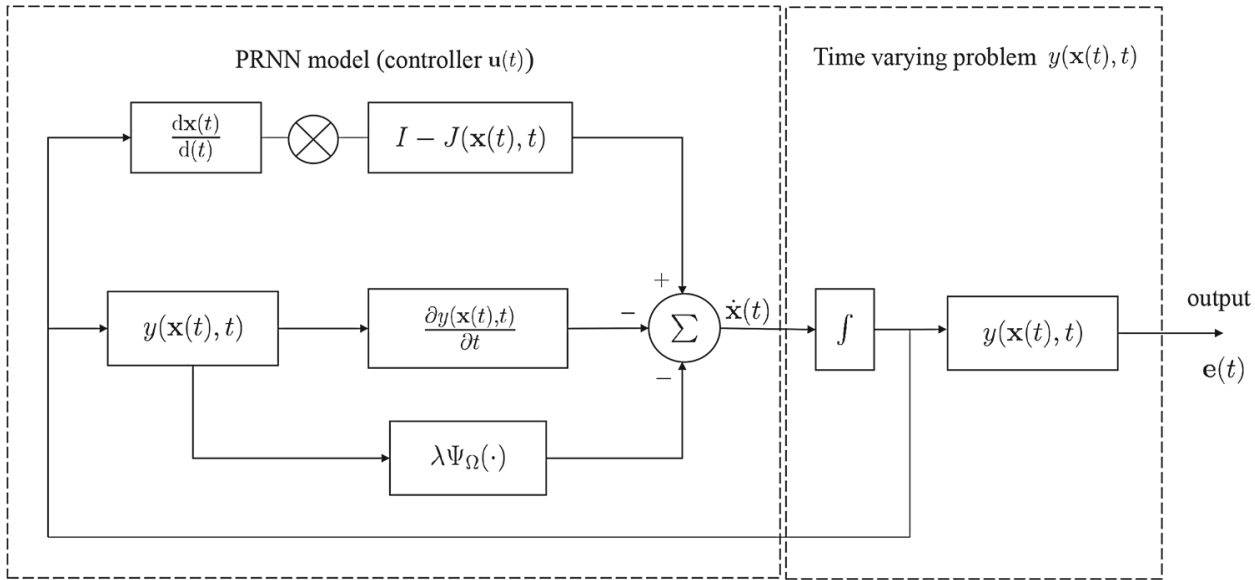

the theoretical solution. The error function and its derivative form are defined as follows:

$$
\begin{aligned}
\mathbf{e}(t) & =y(\mathbf{x}(t), t)-y\left(\mathbf{x}^{*}(t), t\right) \\
& =y(\mathbf{x}(t), t)-\mathbf{0} \\
& =\left[y_{1}(\mathbf{x}(t), t), y_{2}(\mathbf{x}(t), t), \ldots, y_{m}(\mathbf{x}(t), t)\right]^{\mathrm{T}} \in \mathbb{R}^{m}
\end{aligned}
$$

and

$$
\begin{aligned}
\frac{\mathrm{de}(t)}{\mathrm{d} t} & =\frac{\partial y(\mathbf{x}(t), t)}{\partial t}+\frac{\partial y(\mathbf{x}(t), t)}{\partial \mathbf{x}(t)} \frac{\mathrm{d} \mathbf{x}(t)}{\mathrm{d} t} \\
& =\dot{y}_{t}(\mathbf{x}(t), t)+J(\mathbf{x}(t), t) \frac{\mathrm{d} \mathbf{x}(t)}{\mathrm{d} t},
\end{aligned}
$$

of which the superscript ${ }^{\mathrm{T}}$ signifies the transpose operation of a matrix or a vector, and $J(\mathbf{x}(t), t)=\partial y(\mathbf{x}(t), t) / \partial \mathbf{x}(t) \in$ $\mathbb{R}^{m \times m}$. Evidently, $\mathbf{x}(t)$ will converge to the theoretical solution $\mathbf{x}^{*}(t)$ as $\mathbf{e}(t)$ converges to 0 . Combining with the error function of the above time-varying nonlinear equation, the error design formula of the PRNN model is given as follows:

$\dot{\mathbf{e}}(t)=-\Psi(\lambda \mathbf{e}(t))$,

where $\Psi(\cdot)$ is a nonconvex activation function with a saturation constraint, $\dot{\mathbf{e}}(t)=\mathrm{d} \mathbf{e}(t) / \mathrm{d} t$ and $\lambda>0$ is the convergence coefficient. When the error function of the time-varying equation satisfies (4), the state variable $\mathbf{x}(t)$ will exponentially converge to the theoretical solution $\mathbf{x}^{*}(t)$, and the PRNN model is shown as follows:

$\dot{\mathbf{x}}(t)=-J^{-1}\left(\Psi(\lambda y(\mathbf{x}(t), t))+\dot{y}_{t}(\mathbf{x}(t), t)\right)$,

where the symbol ${ }^{-1}$ represents the inversion of a matrix and the block diagram of the presented PRNN model (5) is given from the control perspective [34] and shown in Fig. 1. It is worth noting that $\dot{\mathbf{x}}(t)$ in PRNN model (5) is equivalent to the controller $\mathbf{u}(t)$ in Fig. 1, which drives the output $\mathbf{e}(t)$ to zero with the state variable $\mathbf{x}(t)$ consequently driven to the desired solution $\mathbf{x}^{*}(t)$.

To further debate the robustness of PRNN model (5) in constant noise environment, a formulation is given as

$\dot{\mathbf{x}}(t)=-J^{-1}\left(\Psi(\lambda y(\mathbf{x}(t), t))+\dot{y}_{t}(\mathbf{x}(t), t)\right)+\eta(t)$,

of which $\eta(t) \in \mathbb{R}^{m}$ represents the constant noise. In addition, the traditional RNN model is also provided to facilitate the subsequent analysis and comparison:

$\dot{\mathbf{x}}(t)=-J^{-1}\left(\lambda y(\mathbf{x}(t), t)+\dot{y}_{t}(\mathbf{x}(t), t)\right)$,

which is originated from the error design formula $\dot{\mathbf{e}}(t)=$ $-\lambda \mathbf{e}(t)$ [34].

\section{Theoretical analyses and results}

In this section, the general form of project function is presented, and the global convergence performance of PRNN model (5) is proven.

\section{Formulation of the project function}

Considering the variables $C$ and $\Omega$ with $0 \in \Omega$, the project function is defined as follows $[35,36]$ :

$\Psi_{\Omega}(C)=\arg \min _{A \in \Omega}\|A-C\|_{2}$

wherein $\|\cdot\|_{2}$ denotes the 2-norm of a matrix or a vector.

\section{Global convergence of PRNN model with noise free}

The following theoretical analysis proves the global convergence of PRNN model (5) through mathematical derivations. 
Theorem 1 For the state variable $\mathbf{x}(t)$ in the time-varying nonlinear equations, the global convergence to the theoretical solution can be achieved under any given initial value.

Proof The traditional Lyapunov method [29] is applied to demonstrate the global convergence of PRNN model (5). First, a Lyapunov function candidate is defined as follows:

$\zeta(t)=\mathbf{e}^{\mathrm{T}}(t) \mathbf{e}(t) / 2$.

Evidently, only when $\mathbf{e}(t)=\mathbf{0}, \zeta(t)=0$, and $\zeta(t)>0$ for any $\mathbf{e}(t) \neq \mathbf{0}$. Therefore, it can be concluded that the term of the Lyapunov equation is positive definite. Taking the derivative of both sides of the above equation obtains

$\dot{\zeta}(t)=\mathbf{e}^{\mathrm{T}}(t) \dot{\mathbf{e}}(t)=-\mathbf{e}^{\mathrm{T}}(t) \Psi_{\Omega}(\lambda \mathbf{e}(t))$.

Given $\Psi_{\Omega}(\cdot)$ defined in (8), for all $A \in \Omega$, it can be shown that

$\left\|\Psi_{\Omega}(\lambda \mathbf{e}(t))-\mathbf{e}(t)\right\|_{2}^{2} \leq\|C-\mathbf{e}(t)\|_{2}^{2}$.

Letting $C=0$, the following inequality can be gotten:

$\left\|\Psi_{\Omega}(\lambda \mathbf{e}(t))-\mathbf{e}(t)\right\|_{2}^{2} \leq\|\mathbf{e}(t)\|_{2}^{2}$.

Rearranging the above inequality, we can receive

$\Psi_{\Omega}^{\mathrm{T}}(\lambda \mathbf{e}(t)) \Psi_{\Omega}(\lambda \mathbf{e}(t))-2 \mathbf{e}^{\mathrm{T}}(t) \Psi_{\Omega}(\lambda \mathbf{e}(t)) \leq 0$

The above inequality can be rewritten as

$-\mathbf{e}^{\mathrm{T}}(t) \Psi_{\Omega}(\lambda \mathbf{e}(t)) \leq-\Psi_{\Omega}^{\mathrm{T}}(\lambda \mathbf{e}(t)) \Psi_{\Omega}(\lambda \mathbf{e}(t)) / 2 \leq 0$.

Combining the above equation with (9), it can be concluded that

$\dot{\zeta}(t) \leq-\Psi_{\Omega}^{\mathrm{T}}(\lambda \mathbf{e}(t)) \Psi_{\Omega}(\lambda \mathbf{e}(t)) / 2 \leq 0$.

From the above derived conclusion, the error function can converge to 0 as $t \rightarrow+\infty$. In other words, the state variable $\mathbf{x}(t)$ of the time-varying nonlinear equation can converge to the theoretical solution $\mathbf{x}^{*}(t)$ with a random initial state. The proof is complete.

\section{Global convergence of PRNN model with constant noise}

The following theorem is provided to demonstrate the convergence ability of the proposed PRNN model (5) when confronted with constant noise.

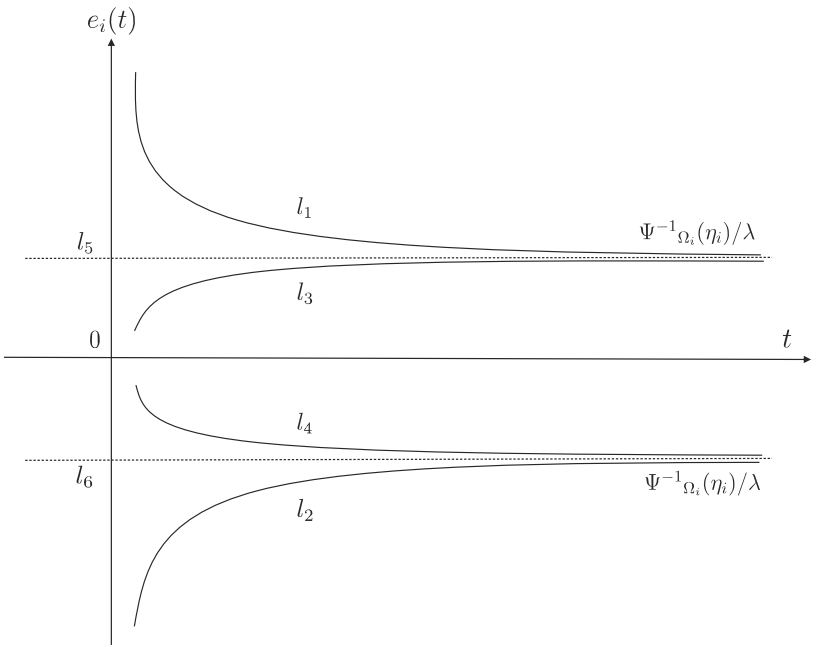

Fig. 2 Trend of $\mathbf{e}_{i}(t)$ in three cases

Theorem 2 For a bounded activation function $\Psi_{\Omega}(\cdot)$ limited by the upper bound $\Psi_{\Omega}^{+}$and lower bound $\Psi_{\Omega}^{-}$under the condition of $0<\eta(t)<\Psi_{\Omega}^{+}$or $0>\eta(t)>\Psi_{\Omega}^{-}$, the residual error of the proposed PRNN model (4) exponentially converges to a bounded value with the interference of constant noise $(t)$.

Proof Introduce the noise term and PRNN model (5) can be redefined as

$\dot{\mathbf{e}}(t)=(t)-\Psi_{\Omega}(\lambda \mathbf{e}(t))$.

Taking the $i$ th subsystem of the above equation, one can obtain

$\dot{e}_{i}(t)=\eta_{i}(t)-\Psi_{\Omega i}\left(\lambda e_{i}(t)\right)$.

Then, define a Lyapunov function candidate as $\rho_{i}(t)=$ $e_{i}^{2}(t) / 2$ and then its time derivative is written as

$\dot{\rho}_{i}(t)=e_{i}(t) \dot{e}_{i}(t)$.

Substituting (11) into (12) generates

$\dot{\rho}_{i}(t)=e_{i}(t)\left(\eta_{i}(t)-\Psi_{\Omega i}\left(\lambda e_{i}(t)\right)\right)$.

The analysis of $\dot{\rho}_{i}(t)$ is summarized as the following three categories: (a) $e_{i}(t)>0$; (b) $e_{i}(t)=0$; (c) $e_{i}(t)<0$. (a) In the case of $e_{i}(t)>0$, it can be easily gotten that $\Psi_{\Omega i}\left(\lambda e_{i}(t)\right)>0$ and the next discussion can be divided into the following three cases: (1) $\Psi_{\Omega i}\left(\lambda e_{i}(t)\right)>\eta_{i}(t)$; (2) $\Psi_{\Omega i}\left(\lambda e_{i}(t)\right)=\eta_{i}(t) ;(3) \Psi_{\Omega i}\left(\lambda e_{i}(t)\right)<\eta_{i}(t)$.

- For case (1), $\Psi_{\Omega i}\left(\lambda e_{i}(t)\right)>\eta_{i}(t)$. Then, it can be generated naturally that $\dot{\rho}_{i}(t)<0$, i.e., $e_{i}(t)$ would decrease with time until $\Psi_{\Omega i}\left(\lambda e_{i}(t)\right)-\eta_{i}(t)=0$, which means 
that $e_{i}(t)$ will finally converge to the bounded line as $l_{1}$ plotted in Fig. 2.

- For case (2), $\Psi_{\Omega i}\left(\lambda e_{i}(t)\right)-\eta_{i}(t)=0$. In this case, system (11) keeps stable at bounded line $l_{5}$ expressed in Fig. 2 and it can be readily inferred that $e_{i}(t)=\Psi_{\Omega i}^{-1}((t)) / \lambda$, where $\Psi_{\Omega i}^{-1}(\cdot)$ reveals the $i$ th subsystem of $\Psi_{\Omega}^{-1}(\cdot)$.

- For case (3), $\lambda \Psi_{\Omega i}\left(e_{i}(t)\right)<\eta_{i}(t)$, that is $\dot{\rho}_{i}(t)>0$. In this situation, $e_{i}(t)$ will finally diverge to the upper limitation of the activation function. If $\lambda \Psi_{\Omega i}^{+} \geq \eta_{i}(t), e_{i}(t)$ will eventually converge to the bounded line $l_{3}$ portrayed in Fig. 2. However, if $\lambda \Psi_{\Omega i}^{+}<\eta_{i}(t)$, the only feasible method is to increase $\lambda$ to ensure the convergence.

(b) In view of $e_{i}(t)=0$, it can be easily obtained $\Psi_{\Omega i}\left(\lambda e_{i}(t)\right)=0$ and $\dot{e}_{i}(t)=\eta_{i}(t)$. In this regard, the change trend of $e_{i}(t)$ is up to the value of $\eta_{i}(t)$, and the state of $e_{i}(t)=0$ is just an instantaneous state value. On the condition that $e_{i}(t)<0$ for $\eta_{i}(t)<0$ or $e_{i}(t)>0$ for $\eta_{i}(t)>0$, the following analysis would accordingly back to the situation (a) $e_{i}(t)>0$ or situation (c) $e_{i}(t)<0$.

(c) With a view to $e_{i}(t)<0$, the discussions are also divided into the following three cases: (1) $\Psi_{\Omega i}\left(\lambda e_{i}(t)\right)<$ $\eta_{i}(t)$; (2) $\Psi_{\Omega i}\left(\lambda e_{i}(t)\right)=\eta_{i}(t)$; (3) $\Psi_{\Omega i}\left(\lambda e_{i}(t)\right)>\eta_{i}(t)$. Since the derivation of the proof is similar to that in case (a), the specific process is not explained in detail. It should be pointed that, unlike in (a), for the initial value of $e_{i}(t)$ less than zero, the corresponding comparison object of case (3) has changed to the lower bound value of $\Psi_{\Omega}^{-}$. However, the final convergence performance is consistent with case (a). As shown in Fig. $2, l_{2}, l_{6}, l_{4}$ are corresponding to the convergence performance of case (1)-(3).

In general, satisfying the condition of $\lambda \Psi_{\Omega}^{-}<\eta(t)<0$ or $\lambda \Psi_{\Omega}^{+}>\eta(t)>0$, it can be derived that $\lim _{t \rightarrow \infty} \mathbf{e}(t)=$ $\Psi_{\Omega}^{-1}((t)) / \lambda$. In other words, the residual error of the nonlinear equation solved by PRNN model (5) can converge to a bounded value under the obstruction of constant noise. The proof is complete.

\section{Numerical simulation experiment}

In what follows, the proposed PRNN model (5) with saturation constraints and traditional RNN model (7) are exerted to solve the special nonlinear equation in the case that the convergence rate is limited.

\section{Time-varying nonlinear equations example}

Now, the following specific nonlinear equation is considered:

$$
\begin{aligned}
& y(\mathbf{x}(t), t) \\
& \quad=\left[\begin{array}{c}
\ln \left(x_{1}(t)\right)-(t+1)^{-1} \\
x_{1}(t) x_{2}(t)-\sin (t) \exp (-t-1) \\
-2+x_{3}(t)+x_{1}{ }^{2}(t)-x_{2}(t) \sin (t) \\
-t+x_{3}(t)+x_{4}(t)+x_{1}{ }^{2}(t)-x_{2}{ }^{2}(t)
\end{array}\right]=\mathbf{0} \in \mathbb{R}^{4},
\end{aligned}
$$

where $x_{1}(t)>0$. To verify the correctness of the solution solved by the proposed PRNN model (5), the theoretical solution $\mathbf{x}^{*}(t)$ is given as below:

$$
\begin{aligned}
\mathbf{x}^{*}(t)= & {\left[\exp \left(\frac{1}{t+1}\right) ; \sin (t) ; 2+\sin ^{2}(t)\right.} \\
& \left.-\exp \left(\frac{2}{t+1}\right) ; t-2\right]
\end{aligned}
$$

In addition, Jacobian matrix $J(\mathbf{x}(t), t)$ in the proposed PRNN model (5) is defined as follows:

$J(\mathbf{x}(t), t)=\left[\begin{array}{cccc}1 / x_{1}(t) & 0 & 0 & 0 \\ x_{2}(t) & x_{1}(t) & 0 & 0 \\ 2 x_{1}(t) & -\sin (t) & 1 & 0 \\ 2 x_{1}(t) & -2 x_{2}(t) & 1 & 1\end{array}\right]$.

\section{Simulation experiment without noise}

In this section, numerical simulation experiments are performed to verify the superiority convergence of PRNN model (5) with saturation constraints compared with the traditional RNN model (7) in the absence of noise. The initial state variable is set as $\mathbf{x}(0)=[1,1,1,1]^{\mathrm{T}}$, which makes the initial solution error of both RNN model (7) and PRNN model (5) be around 10. Besides, to limit the error convergence rate of the two models to 5, the convergence coefficient of RNN model (7) is set as $\lambda=0.5$, the saturation boundary of PRNN model (5) is limited to 5 , and the convergence coefficient of PRNN model (5) is set as 10. Additionally, the special saturation constraint involved in PRNN model (5) is constructed as follows:

$$
\Psi\left(\lambda e_{i}(t)\right)= \begin{cases}-5 & \lambda e_{i}(t)<-5 \\ e_{i}(t) & -5 \leq \lambda e_{i}(t) \leq 5 \\ 5 & \lambda e_{i}(t)>5\end{cases}
$$

As is seen from Fig. 3, when the error convergence rate is limited to 5, the error between the solution of the traditional RNN model (7) and the theoretical solution can converge to the order of $10^{-3} \sim 10^{-2}$. In contrast, the error between the solution of the PRNN model (5) with saturation constraints and the theoretical solution can reach to a bound with the order of $10^{-4}$ in about $2 \mathrm{~s}$. Therefore, it can be concluded that PRNN model (5) with saturation constraints obviously 


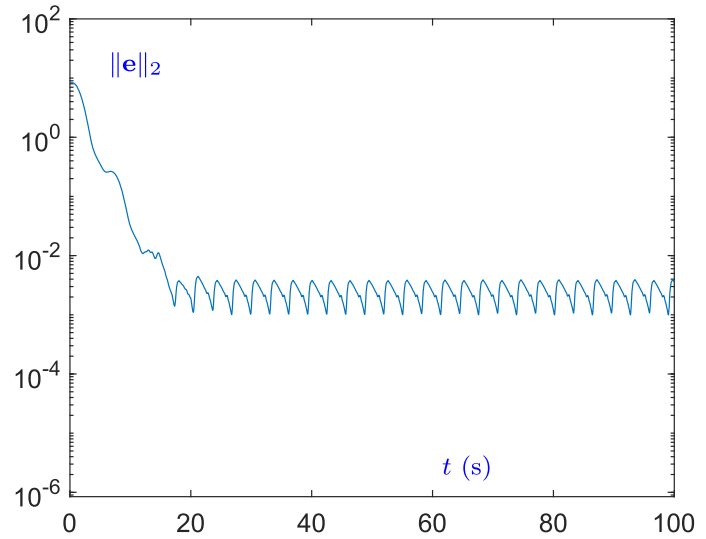

(a)

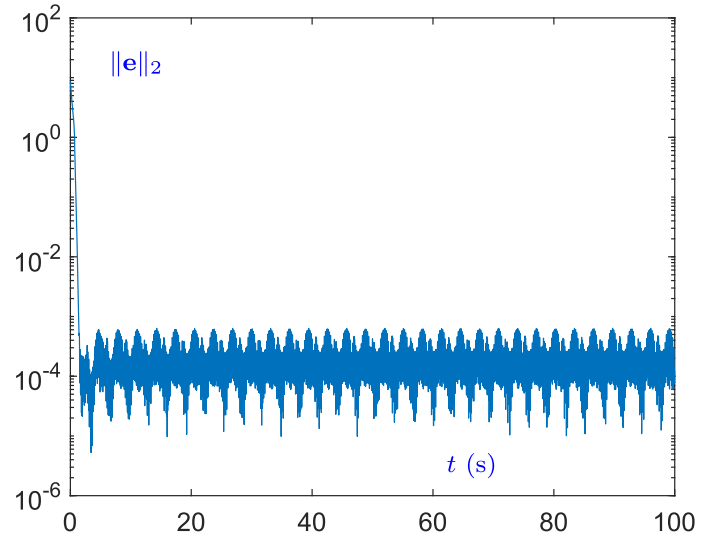

(b)

Fig. 3 The tracking errors of RNN model and PRNN model in solving Eq. (13) with noise free. a RNN model (7). b PRNN model (5)

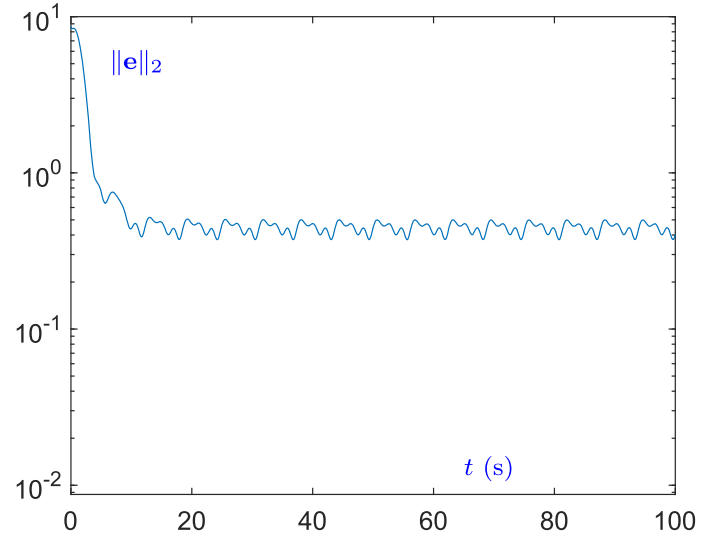

(a)

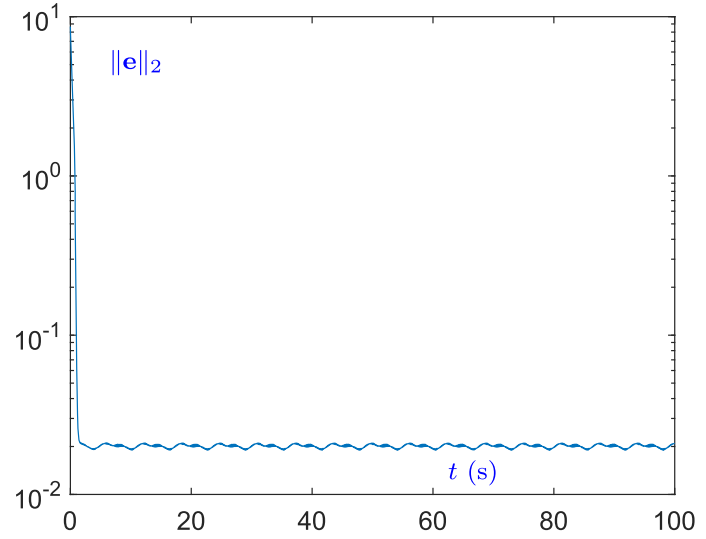

(b)

Fig. 4 The tracking errors of RNN model and PRNN model in solving equation (13) with constant noise $\eta(t)=[0.1,0.1,0.1,0.1]^{\mathrm{T}}$. a RNN model (7). b PRNN model (5)

has a superior convergence performance compared to the traditional RNN model (7) when the convergence rate is limited.

\section{Simulation experiment in front of constant noise}

In this section, under the interference of constant noise, the numerical simulation experiments are conducted to demonstrate the superior robustness of PRNN model (5) with saturation constraints compared with the traditional RNN model (7). The constant noise is set as $\eta(t)=[0.1,0.1,0.1,0.1]^{\mathrm{T}}$.

It can be seen from Fig. 4 that in the face of constant noise, the tracking errors of traditional RNN model (7) need about $10 \mathrm{~s}$ to reach the theoretical solution at an order of $10^{-1} \sim 10^{0}$, while errors between the solution of PRNN model (5) with saturation constraints and the theoretical solution can reach to a bound with the order of $10^{-2} \sim 10^{-1}$ in approximately $2 \mathrm{~s}$ in the presence of constant noise.

\section{PRNN model for intention identification}

In this section, combined with PRNN model (5), the principle of the closed-loop prediction system for the whole lower limb joint movement intention recognition is introduced and the corresponding principle flow chart is given.

\section{Intention identification principle process}

The schematic diagram of lower limb movement intention recognition is provided in Fig. 5. First, the input sEMG signal is converted into muscle activation signal through preprocessing [16,37], and the value of joint torque can be obtained through the HMM $[10,12,15]$. Then, through the Lagrange dynamic model [38], the kinetic energy formula and potential energy formula, the SSHMM concerning the lower limb joint angle is acquired [11], meanwhile introducing the control law to compensate the unmeasured moment muscle group in SSHMM. Finally, through the error function 
Fig. 5 Movement intention recognition process

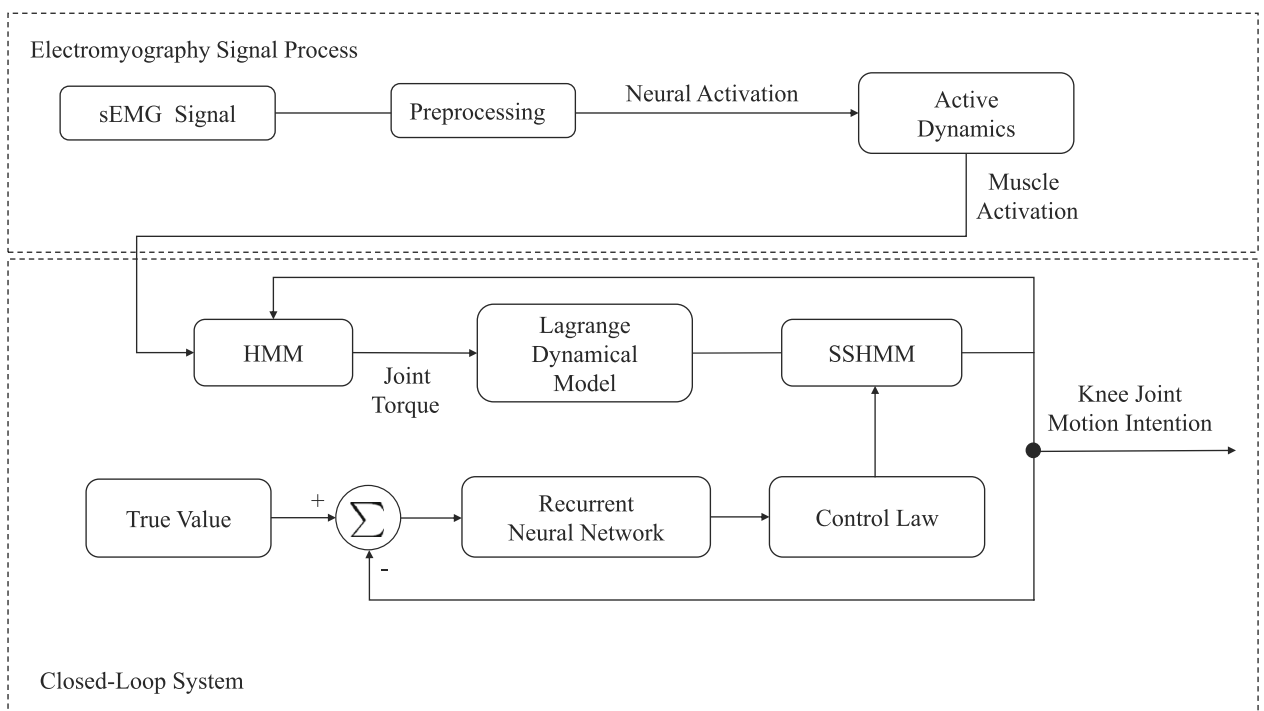

between the true value measured by inertial measurement unit (IMU) and the estimated one, PRNN model (5) is introduced to realize the iteration of the control law. So far, the whole prediction process forms a closed-loop system, and the defined error function can realize a global exponential convergence.

\section{Hill-based muscle model}

Through muscle activation signals, the lower limb joint torque can be calculated using the HMM [12,14,15].

$T=r \cdot\left(F_{1}^{\mathrm{m}}+F_{2}^{\mathrm{m}}\right)=T(\theta(t), \varsigma(t))$,

where $T$ is torque; $r$ is a constant; $F_{1}^{\mathrm{m}}$ is active contraction; $F_{2}^{\mathrm{m}}$ is passive force; $\varsigma(k)$ is the muscle activation; $\theta(t)$ is the value of joint angle.

\section{Lagrange dynamic model}

Utilizing the kinetic energy and potential formula, the following relational equations can be obtained as

$K=\frac{1}{2} I \dot{\theta}^{2}$

$P=-m g \cdot 0.441 l \sin \theta$,

where $K$ is the kinetic energy; $P$ is the gravity potential energy on the shank about knee joint; $l$ is the length of shank and $0.441 l$ is the distance between the center of mass of the shank and the knee joint [39]; $m$ is the mass of the shank; $I, g$ represent the moment of inertia and the gravitational acceleration, respectively. Then, through the Lagrange equation, the relationship between joint torque, lower limb kinetic energy and potential energy is shown as follows:
$L=K-P$

$T=\frac{\partial}{\partial t}\left(\frac{\partial L}{\partial \dot{\theta}}\right)-\frac{\partial L}{\partial \theta}$,

where $L$ is a Lagrangian function. Supposing that $\theta_{1}=\theta$, $\theta_{2}=\dot{\theta}$ and combining equation (17) and (18), one can get

$\dot{\theta}_{1}=\theta_{2}$

$\dot{\theta}_{2}=\frac{1}{I}\left(T+m g \cdot 0.441 l \cos \theta_{1}\right)$.

Letting

$$
\begin{aligned}
\mathbf{f}(\boldsymbol{\theta}, t) & =[\dot{\theta}, m g \cdot 0.441 l \cdot \theta / I]^{\mathrm{T}} \\
\mathbf{g}(T(\theta, \varsigma)) & =[0, T(\theta, \varsigma) / I]^{\mathrm{T}},
\end{aligned}
$$

the SSHMM can be rewritten as below:

$$
\left\{\begin{array}{l}
\dot{\boldsymbol{\theta}}=\mathbf{f}(\boldsymbol{\theta}, t)+\mathbf{g}(T(\theta, \varsigma)) \\
\mathbf{y}=\boldsymbol{\theta}
\end{array}\right.
$$

where $\boldsymbol{\theta}=[\theta, \dot{\theta}]^{\mathrm{T}}$ is the time-varying state variable; $T(\theta, \varsigma)$ is the joint torque calculated by HMM (16). It is worth noting that the joint angle of lower limb can be directly predicted by solving SSHMM (21). However, it may lead to the accumulation of errors, which will cause the estimated value of SSHMM (21) to diverge from the true value measured by IMU as shown in Fig. 6. Due to the parameter estimation in the HMM model and the lack of other unmeasured muscle groups torque in the shank, a more accurate SSHMM is defined as follows by introducing a compensation item $\mathbf{u}(t)$, i.e., control law,

$\left\{\begin{array}{l}\dot{\boldsymbol{\theta}}=\mathbf{f}(\boldsymbol{\theta}, t)+\mathbf{g}(T(\theta, \varsigma))+\mathbf{u}(t) \\ \mathbf{y}=\boldsymbol{\theta}\end{array}\right.$ 


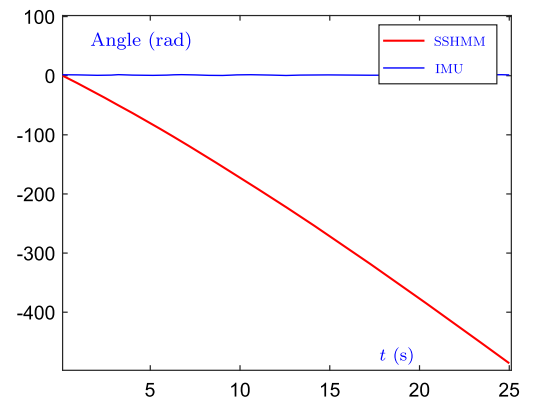

(a)

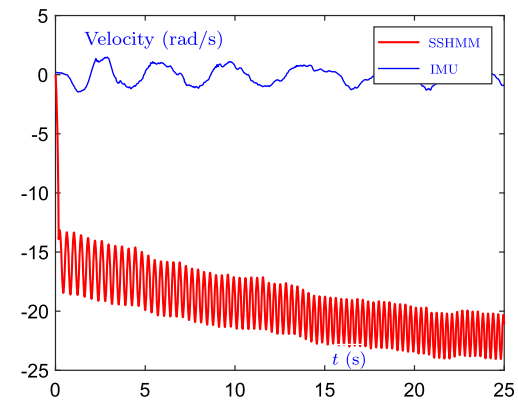

(b)
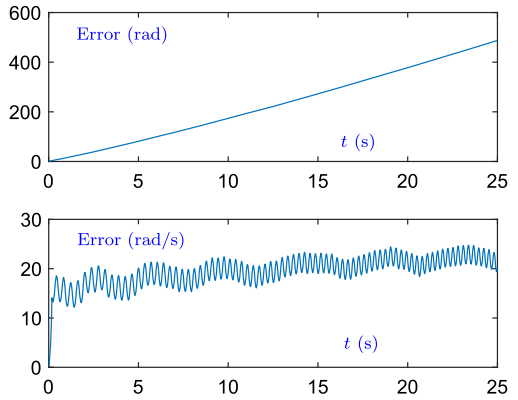

(c)

Fig. 6 The joint angle and joint angular velocity of lower limb estimated by SSHMM (21) compared with the true value measured by IMU. a Joint angle. b Joint angular velocity. c Residual errors

\section{Closed-loop control system}

Exploiting equation (4), and constructing the error function $\mathbf{e}(t)=\boldsymbol{\theta}^{\mathrm{d}}-\boldsymbol{\theta}$, where $\boldsymbol{\theta}^{\mathrm{d}}$ is the desired state measured by IMU, the new control law can be generalized as

$\mathbf{u}_{\mathrm{PRNN}}(t)=\dot{\boldsymbol{\theta}}^{\mathrm{d}}+\Psi\left(\lambda\left(\boldsymbol{\theta}^{\mathrm{d}}-\boldsymbol{\theta}\right)\right)-\mathbf{f}(\boldsymbol{\theta}, t)-\hat{\mathbf{g}}(T(\theta, \varsigma))$,

where $\mathbf{g}(T(\theta, \varsigma))$ of the HMM can be estimated by $\hat{\mathbf{g}}(T(\theta, \varsigma))$. The introduction of the PRNN model (5) allows the control law to be continuously adjusted according to the theoretical value at the current time, thus avoiding the error accumulation and enabling the SSHMM (21) to achieve more accurate estimation.

Remark 1 Some uncertainties in the construction of HMM, such as model simplification and uncalibrated parameters, will affect the accuracy of prediction. In this paper, a classical radial basis function neural network which can estimate any nonlinear function is utilized to estimate $\mathbf{g}(T(\theta, \varsigma))$ in the control law.

Besides, the concepts of $\mathbf{u}(t)$ and $\mathbf{u}_{\mathrm{PRNN}}(t)$ respectively mentioned in (22) and (23) are distinguished in the Remark 2.

Remark $2 \mathbf{u}(t)$ in (22) represents a macroscopic compensation for the torque generated by the unmeasured muscle group of SSHMM (21), while $\mathbf{u}_{\mathrm{PRNN}}(t)$ in (23) represents a specific compensation (i.e., control law) to model SSHMM (21), which is generated by introducing PRNN model (5) to form a closed-loop system. Through the control law $\mathbf{u}_{\mathrm{PRNN}}(t)$, SSHMM (21) can effectively avoid the error accumulation and achieve better prediction of the intention of lower limb joint movement.

\section{Simulation experiment}

In this section, the proposed control method is applied to an actual experimental simulation of lower limb rehabilitation

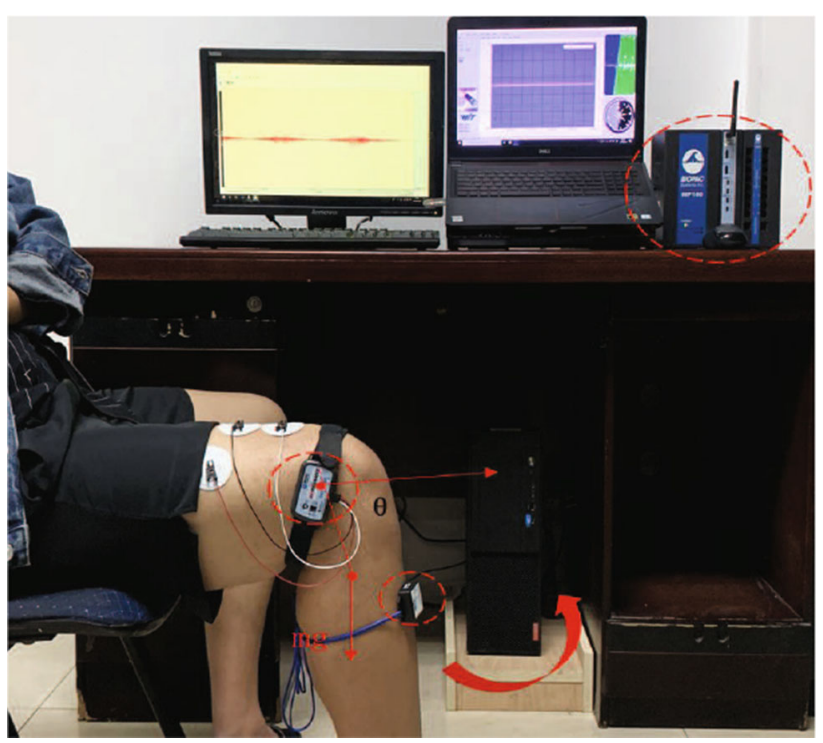

Fig. 7 Actual experiment diagram

robot movement intention recognition to prove its effectiveness and superiority. The height and mass of the subject are $170 \mathrm{~cm}$ and $76 \mathrm{~kg}$, respectively, and the specific experimental process is given as follows. Firstly, clean the rectus femoris muscle with alcohol, and then place three surface electrodes on the rectus femoris muscle to collect sEMG signals. Next, stick the IMU on the lower leg to record the angle and angular velocity of the joints of the lower limbs during the actual exercise. In addition, to allow muscles to be fully exerted during the entire exercise, an external load of $2 \mathrm{~kg}$ is added to the shank of subject. Finally, the subject sits in a chair swinging his legs freely and the knee extension data are recorded during the experiment as shown in Fig. 7. In the experiment, the sEMG signal and the true joint angle measured by IMU are used as the core input for the prediction of the motion intention, and the predicted value of the joint angle of human lower limb is obtained through PRNN model (5). The initial state variable $\boldsymbol{\theta}(0)$ is set as $\boldsymbol{\theta}(0)=[-\pi / 4,50]^{\mathrm{T}}$, and the convergence coefficient of RNN model (7) is set as 2 , which 


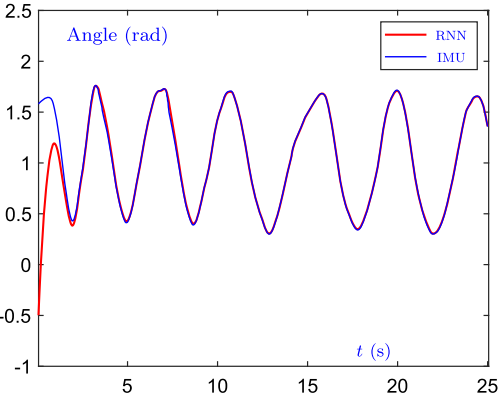

(a)

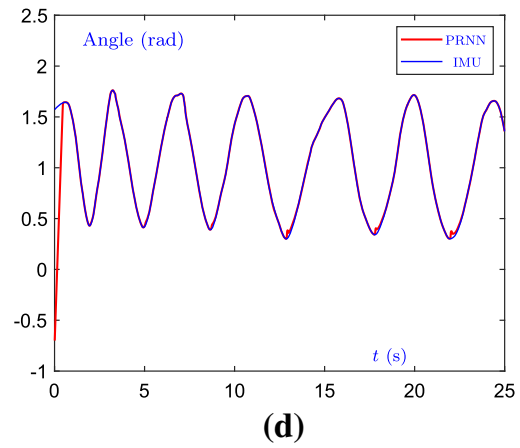

Fig. 8 The joint angle and joint angular velocity of lower limb estimated by SSHMM (22) equipped with RNN model (7) and PRNN model (5) compared with the true value measured by IMU in noise free condition. a Joint angle aided by RNN model (7). b Joint angular velocity

makes sure that the convergence rate of joint angle and joint angular velocity is limited to 5 and 100 , respectively. Similarly, the saturation constraint employed in PRNN model (5) is designed as follows:

$\Psi\left(\lambda e_{1}(t), \lambda e_{2}(t)\right)= \begin{cases}-5 & \lambda e_{1}(t)<-5 \\ \lambda e_{1}(t) & -5 \leq \lambda e_{1}(t) \leq 5 \\ 5 & \lambda e_{1}(t)>5, \\ -100 & \lambda e_{2}(t)<-100 \\ \lambda e_{2}(t) & -100 \leq \lambda e_{2}(t) \leq 100 \\ 100 & \lambda e_{2}(t)>100,\end{cases}$

which also ensures that the error convergence rates of joint angle and joint angular velocity are limited to 5 and 100 , respectively. Besides, the convergence coefficient of PRNN model (5) is set as 100 , and the duration of the whole experiment is set to $25 \mathrm{~s}$.

In the case of limited error convergence rate, Fig. 8 shows simulation results of the SSHMM (22) equipped with the traditional RNN model (7) and PRNN model (5) with saturation constraints in the condition of noise free. Compared with the estimation through SSHMM (21) directly in Fig. 6, SSHMM (22) with traditional RNN model (7) and PRNN model (5) successfully avoids the divergence of the esti-
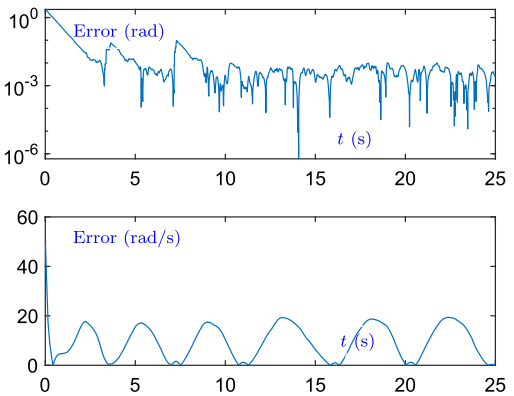

(c)
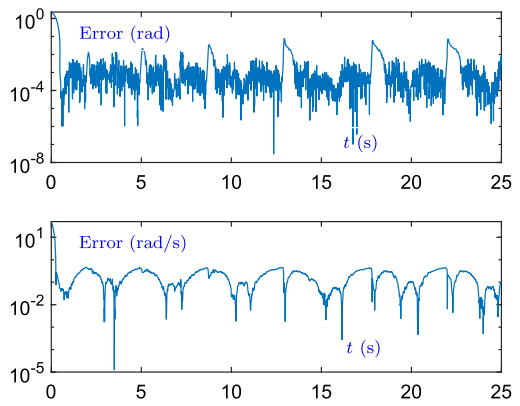

(f)

aided by RNN model (7). c Residual errors aided by RNN model (7). d Joint angle aided by PRNN model (5). e Joint angular velocity aided by PRNN model (5). f Residual errors aided by PRNN model (5)

mated values, which demonstrates the effectiveness of the closed-loop framework. As performed in Fig. 8a, d, the estimated joint angle of RNN model (7) and PRNN model (5) can steadily track the true value measured by IMU, while the estimated joint angular velocity of RNN model (7) always has a large deviation from the true value measured by IMU compared with PRNN model (5) as shown in Fig. 8b, e. Additionally, the convergence performance of RNN model (7) and PRNN model (5) is clearly shown in Fig. 8c, f. Among them, it can be seen from Fig. $8 \mathrm{c}$ that the absolute tracking error of joint angle always presents a downward trend and reaches to a bound with the order of $10^{-4} \sim 10^{-2}$ within 10 $\mathrm{s}$, while the tracking error of joint angular velocity always maintains a large gap with the measured value. In contrast, the absolute tracking error of joint angle rapidly converges to $10^{-4} \sim 10^{-2}$ within $0.5 \mathrm{~s}$, and the tracking error of joint angular velocity converges to a bound and remains at an order of $10^{-3} \sim 10^{-2}$ within $0.5 \mathrm{~s}$. Through the analysis of the above experimental results, one can conclude that SSHMM (22) equipped with PRNN model (5) obviously has better convergence performance under the condition of limited convergence rate compared with SSHMM (22) with traditional RNN model (7). 


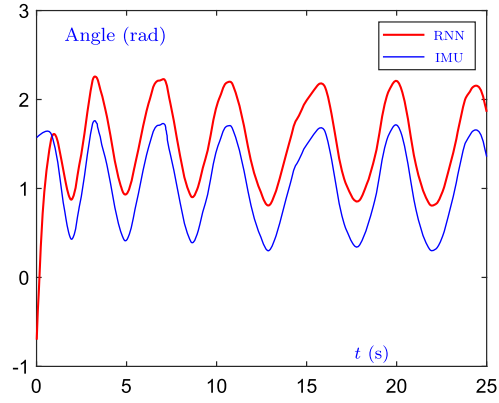

(a)

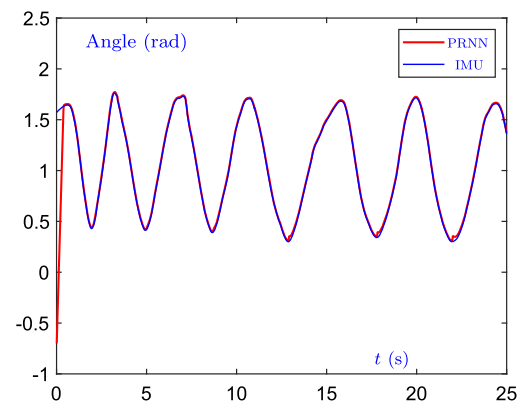

(d)

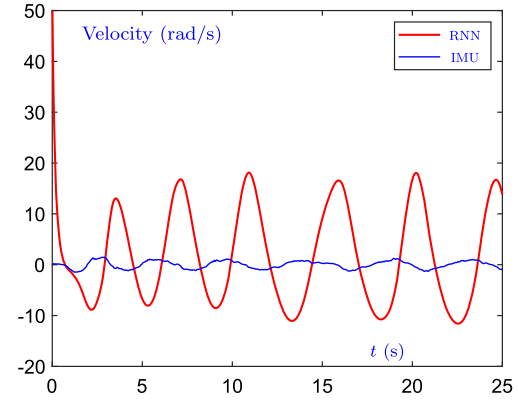

(b)

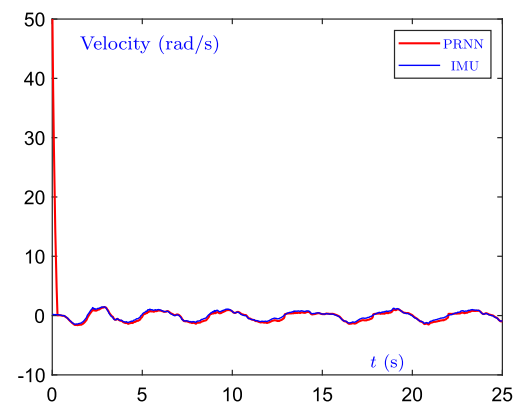

(e)
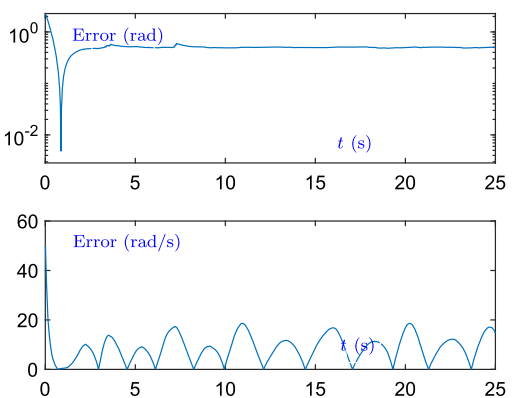

(c)
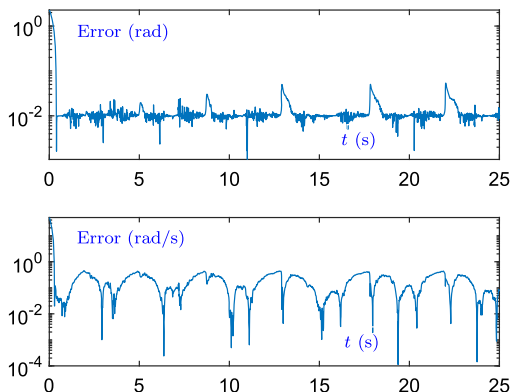

(f)
Fig. 9 The joint angle and joint angular velocity of lower limb estimated by SSHMM (22) equipped with RNN model (7) and PRNN model (5) compared with the true value measured by IMU in the presence of constant noise $\omega(t)=[1,1]^{\mathrm{T}}$. a Joint angle aided by RNN model (7). b
Joint angular velocity aided by RNN model (7). $\mathbf{c}$ Residual errors aided by RNN model (7). d Joint angle aided by PRNN model (5). e Joint angular velocity aided by PRNN model (5). f Residual errors aided by PRNN model (5)
In addition, the SSHMM (21) with noise disturbance is defined as follows:

$$
\left\{\begin{array}{l}
\dot{\boldsymbol{\theta}}=\mathbf{f}(\boldsymbol{\theta}, t)+\mathbf{g}(T(\theta, \varsigma))+\omega(t) \\
\mathbf{y}=\boldsymbol{\theta}
\end{array}\right.
$$

where $\omega(t)=[1,1]^{\mathrm{T}}$ represents the constant noise. As can be seen from Fig. 9a, d, under the interference of constant noise, SSHMM (22) synthesized RNN model (7) and PRNN model (5) can still well estimate the joint angles in the process of lower limb movement, while traditional RNN model (7) without the saturation constraint cannot make SSHMM (22) successfully predict the joint angular velocity in the process of lower limb movement, as shown in Fig. 9b. Besides, it can be seen intuitively in Fig. 9c that the absolute tracking error of joint angle by the SSHMM (22) with RNN model (7) achieves stable convergence with the order of $10^{-1} \sim 10^{0}$ in about $3 \mathrm{~s}$. However, there is an oscillation error with a peak value of 20 between the estimated value of joint angular velocity during movement and the true value measured by the IMU. On the other hand, the absolute tracking error of joint angle rapidly converges to a bound and remains at an order of $10^{-2}$ within $0.5 \mathrm{~s}$, and the absolute tracking error of joint angular velocity reaches to a bound with the order of $10^{-3} \sim 10^{-1}$ within $0.5 \mathrm{~s}$. By analyzing the above experimental results, one can summarize that under the condition of constant noise interference, SSHMM (22) with the saturation-constrained PRNN model (5) can preferably estimate the joint angle and joint angular velocity during the movement of the lower limb, compared with the traditional RNN model (7) without the saturation constraint.

\section{Conclusions}

In this paper, the PRNN model with the saturation constraint and the traditional RNN model have been researched under the condition that the convergence rate of the model is limited. The derivation process of PRNN model has been given by combining the time-varying nonlinear equation in a general form. Besides, through the Lyapunov stability theorem, strict theoretical proofs and analysis have been given for the convergence and robustness of the PRNN model. To demonstrate the superiority of PRNN model with the saturation constraint, comparative numerical simulations and 
experiments on the lower limb rehabilitation robot intention recognition synthesized by the proposed PRNN model with saturation constraints and the traditional RNN model have been conducted.

Acknowledgements This study is funded by the Natural Science Foundation of Chongqing (China) under Grant cstc2020jcyj-zdxmX0028, by the CAS "Light of West China" Program, and by the Chongqing Science and Technology Bureau under grant cstc2018jszx-cyzdX0041.

\section{Declarations}

Conflict of interest The authors declare that they have no conflict of interest.

Open Access This article is licensed under a Creative Commons Attribution 4.0 International License, which permits use, sharing, adaptation, distribution and reproduction in any medium or format, as long as you give appropriate credit to the original author(s) and the source, provide a link to the Creative Commons licence, and indicate if changes were made. The images or other third party material in this article are included in the article's Creative Commons licence, unless indicated otherwise in a credit line to the material. If material is not included in the article's Creative Commons licence and your intended use is not permitted by statutory regulation or exceeds the permitted use, you will need to obtain permission directly from the copyright holder. To view a copy of this licence, visit http://creativecomm ons.org/licenses/by/4.0/.

\section{References}

1. Yang C, Peng G, Li Y, Cui R, Cheng L, Li Z (2019) Neural networks enhanced adaptive admittance control of optimized robotenvironment interaction. IEEE Trans Cybern 49(7):2568-2579

2. Xie Z, Jin L, Du X, Xiao X, Li H, Li S (2019) On generalized RMP scheme for redundant robot manipulators aided with dynamic neural networks and nonconvex bound constraints. IEEE Trans Ind Inf 15(9):5172-5181

3. Li Q, Kroemer O, Su Z, Veiga FF, Kaboli M, Ritter H (2020) A review of tactile information: perception and action through touch. IEEE Trans Robot 36(6):1619-1634

4. Chen T, Chiu MC (2021) An interval fuzzy number-based fuzzy collaborative forecasting approach for DRAM yield forecasting. Complex Intell Syst 7(2):111-122. https://doi.org/10.1007/ s40747-020-00179-8

5. Alfarraj O, Tolba A (2020) A two-level computer vision-based information processing method for improving the performance of human-machine interaction-aided applications. Complex Intell Syst. https://doi.org/10.1007/s40747-020-00208-6

6. Yang C, Chen C, He W, Cui R, Li Z (2019) Robot learning system based on adaptive neural control and dynamic movement primitives. IEEE Trans Neural Netw Learn Syst 30(3):777-787

7. Geib CW, Goldman RP (2009) A probabilistic plan recognition algorithm based on plan tree grammars. Artif Intell 173(11):11011132

8. Anh HT, Pereira LM, Santos F (2011) The role of intention recognition in the evolution of cooperative behavior. AAAI, Palo Alto

9. Sadri F (2011) Logic-based approaches to intention recognition. Handbook of research on ambient intelligence and smart (environments)
10. Ding Q, Xiong A, Zhao X, Han J (2011) A novel EMG-driven state space model for the estimation of continuous joint movements. In: Proc. IEEE Int. Conf. Syst., Man, Cybern. Anchorage, AK 28912897

11. Duan F, Dai L (2017) Recognizing the gradual changes in sEMG characteristics based on incremental learning of wavelet neural network ensemble. IEEE Trans Ind Electron 64(5):4276-4286

12. Duan F, Dai L, Chang W, Chen Z, Zhu C, Li W (2016) SEMG-based identification of hand motion commands using wavelet neural network combined with discrete wavelet transform. IEEE Trans Ind Electron 63(3): 1923-1934

13. Buchanan TS, Lloyd DG, Manal K, Besier TF (2004) Neuromusculoskeletal modeling: estimation of muscle forces and joint moments and movements from measurements of neural command. J Appl Biomech 20(4):367-395

14. Fleischer C, Hommel G (2007) Calibration of an EMG-based body model with six muscles to control a leg exoskeleton. In: Proc. IEEE Int. Conf. Robot. Autom., pp 2514-2519

15. Fleischer C, Hommel G (2008) A human-exoskeleton interface utilizing electromyography. IEEE Trans Robot 24(4):872-882

16. Han J, Ding Q, Xiong A, Zhao X (2015) A state-space EMG model for the estimation of continuous joint movements. IEEE Trans Ind Electron 62(7):4267-4375

17. Luo X, Zhou M, Li S, Xia Y, You Z, Zhu Q, Leung H (2018) Incorporation of efficient second-order solvers into latent factor models for accurate prediction of missing QoS data. IEEE Trans Cybern 48(4):1216-1228

18. Yu H (2020) Evaluation of cloud computing resource scheduling based on improved optimization algorithm. Complex Intell Syst. https://doi.org/10.1007/s40747-020-00163-2

19. Yang C, Chen C, Wang N, Ju Z, Fu J, Wang M (2019) Biologically inspired motion modeling and neural control for robot learning from demonstrations. IEEE Trans Cogn Dev Syst 11(2):281-291

20. Luo J, Yang C, Li Q, Wang M (2019) A task learning mechanism for the telerobots. Int J Hum Robot 16(2):1950009

21. Wen X, Qin S (2021) A projection-based continuous-time algorithm for distributed optimization over multi-agent systems. Complex Intell Syst. https://doi.org/10.1007/s40747-020-00265-x

22. Qi Y, Jin L, Wang Y, Xiao L, Zhang J (2020) Complex-valued discrete-time neural dynamics for perturbed time-dependent complex quadratic programming with applications. IEEE Trans Neural Netw Learn Syst 31(9):3555-3569

23. Bao G, Zhang Y, Zeng Z (2020) Memory analysis for memristors and memristive recurrent neural networks. IEEE/CAA J Autom Sin 7(1):96-105

24. Jin J (2021) A robust zeroing neural network for solving dynamic nonlinear equations and its application to kinematic control of mobile manipulator. Complex Intell Syst 7(3):87-99. https://doi. org/10.1007/s40747-020-00178-9

25. Luo Y, Zhao S, Yang D, Zhang H (2020) A new robust adaptive neural network backstepping control for single machine infinite power system with TCSC. IEEE/CAA J Autom Sin 7(1):48-56

26. Wei L, Jin L, Yang C, Chen K, Li W (2019) New noise-tolerant neural algorithms for future dynamic nonlinear optimization with estimation on Hessian matrix inversion. IEEE Trans Syst Man Cybern. https://doi.org/10.1109/TSMC.2019.2916892 (in press)

27. Xie Z, Jin L, Luo X, Sun Z, Liu M (2020) RNN for repetitive motion generation of redundant robot manipulators: An orthogonal projection based. IEEE Trans Neural Netw Learn Syst. https://doi. org/10.1109/TNNLS.2020.3028304 (in press)

28. Xie Z, Jin L, Luo X, Li S, Xiao X (2021) A data-driven cyclicmotion generation scheme for kinematic control of redundant manipulators. IEEE Trans Contr Syst Technol 29(1):53-63

29. Jin L, Liufu Y, Lu H, Zhang Z (2020) Saturation-allowed neural dynamics applied to perturbed time-dependent system of linear 
equations and robots. IEEE Trans Ind Electron. https://doi.org/10. 1109/TIE.2020.3029478 (in press)

30. Jin L, Yan J, Du X, Xiao X, Fu D (2020) RNN for solving time-variant generalized Sylvester equation with applications to robots and acoustic source localization. IEEE Trans Ind Inform 16(10):6359-6369

31. Sun Z, Sun Y, Li Y, Liu K (2019) A New trust region-sequential quadratic programming approach for nonlinear systems based on nonlinear model predictive control. Eng Optim 51(6):1071-1096

32. Qi Y, Jin L, Luo X, Zhou M (2020) Recurrent neural dynamics models for perturbed nonstationary quadratic programs: A control-theoretical perspective. IEEE Trans Neural Netw Learn Syst. https://doi.org/10.1109/TNNLS.2020.3041364 (in press)

33. Sun Z, Shi T, Wei L, Sun Y, Liu K, Jin L (2020) Noise-suppressing zeroing neural network for online time-varying nonlinear optimization problems: A control-based approach. Neural Comput Appl 32(2):11505-11520

34. Jin L, Zhang Y, Li S, Zhang Y (2017) Noise-tolerant ZNN models for solving time-varying zero-finding problems: a control-theoretic approach. IEEE Trans Autom Control 62(2):992-997
35. Cheng L, Liu W, Hou ZG, Huang T, Yu J, Tan M (2017) An adaptive Takagi-Sugeno model based fuzzy predictive controller for piezoelectric actuators. IEEE Trans Ind Electron 64(4):3048-3058

36. Luo X, Sun J, Wang Z, Li S, Shang M (2017) Symmetric and nonnegative latent factor models for undirected, high-dimensional, and sparse networks in industrial applications. IEEE Trans Ind Inform 13(6):3098-3107

37. Lloyd DG, Bessier TE (2003) An EMG-driven musculoskeletal model to estimate muscle forces and keen joint moments in vivo. J Biomech 36(6):765-776

38. Han SI, Lee JM (2017) Approximation-free tracking error constraint control for Lagrangian systems. IEEE Trans Ind Electron 64(2):1269-1278

39. Jin DW, Zhang JC, Wang RC (2011) Bio-mechanology in rehabilitation engineering. Tsinghua University, Beijing

Publisher's Note Springer Nature remains neutral with regard to jurisdictional claims in published maps and institutional affiliations. 